\title{
Whole-Genome Expression Analysis and Signal Pathway Screening of Synovium-Derived Mesenchymal Stromal Cells in Rheumatoid Arthritis
}

\author{
Jingyi Hou, Yi Ouyang, Haiquan Deng, Zhong Chen, Bin Song, Zhongyu Xie, \\ Peng Wang, Jinteng Li, Weiping Li, and Rui Yang
}

Department of Orthopedics, Sun Yat-sen Memorial Hospital, Sun Yat-sen University, 107 Yan Jiang Road West, Guangzhou 510120, China

Correspondence should be addressed to Weiping Li; lwp63@163.com and Rui Yang; yangr@mail.sysu.edu.cn

Received 8 April 2016; Revised 25 June 2016; Accepted 29 June 2016

Academic Editor: Armand Keating

Copyright (C) 2016 Jingyi Hou et al. This is an open access article distributed under the Creative Commons Attribution License, which permits unrestricted use, distribution, and reproduction in any medium, provided the original work is properly cited.

\begin{abstract}
Synovium-derived mesenchymal stromal cells (SMSCs) may play an important role in the pathogenesis of rheumatoid arthritis (RA) and show promise for therapeutic applications in RA. In this study, a whole-genome microarray analysis was used to detect differential gene expression in SMSCs from RA patients and healthy donors (HDs). Our results showed that there were 4828 differentially expressed genes in the RA group compared to the HD group; 3117 genes were upregulated, and 1711 genes were downregulated. A Gene Ontology analysis showed significantly enriched terms of differentially expressed genes in the biological process, cellular component, and molecular function domains. A Kyoto Encyclopedia of Genes and Genomes analysis showed that the MAPK signaling and rheumatoid arthritis pathways were upregulated and that the p53 signaling pathway was downregulated in RA SMSCs. Quantitative real-time polymerase chain reaction was applied to verify the expression variations of the partial genes mentioned above, and a western blot analysis was used to determine the expression levels of p53, p-JNK, p-ERK, and p-p38. Our study found that differentially expressed genes in the MAPK signaling, rheumatoid arthritis, and p53 signaling pathways may help to explain the pathogenic mechanism of RA and lead to therapeutic RA SMSC applications.
\end{abstract}

\section{Introduction}

Rheumatoid arthritis (RA) is considered a chronic, autoimmune joint disease characterized by systemic inflammation, autoantibodies, and synovitis with an unclear etiology [1]. Disease-modifying antirheumatic drugs (DMARDs) are conventional, key therapeutic agents that have been used to reduce systemic inflammation and synovitis [2]. When arthritis is uncontrolled or when DMARDs result in toxic effects, biological agents, for example, tumor necrosis factor (TNF) inhibitors and agents targeting the interleukin- (IL-) 1 and IL6 pathways, T-cell costimulatory pathways, and B cells will be used [3]. However, DMARDs do not effectively prevent cartilage damage, and the effects of biological agents remain largely unclear. Thus, it is necessary to develop new RA treatment methods.

Mesenchymal stromal cells (MSCs) are nonhematopoietic, multipotent stem cells that have been isolated and characterized from many human tissues, including bone marrow, synovium, adipose, and muscle [4]. As MSCs have an immunosuppressive property and are capable of multipotent differentiation, they have been widely applied for repairing tissue and treating immune disorders [5]. Synovium-derived mesenchymal stromal cells (SMSCs) not only have the immunosuppressive property and multipotent differentiation ability as other MSCs but also possess a greater ability for chondrogenesis [6], which could be very beneficial for repairing the cartilage damage caused by RA. Moreover, allogenic MSC transplantations could potentially be rejected or induce apoptosis in the allogeneic stem cells [7-9]; therefore, autologous SMSC treatments have massive potential for RA patients. However, the biological characteristics of RA SMSCs are largely unknown.

The aim of this study was to identify differentially expressed genes of SMSCs from the synovial tissue of RA patients and healthy donors (HDs). We found that there 
TABLE 1: Demographics and characteristics of rheumatoid arthritis (RA) patients and healthy donors (HDs).

\begin{tabular}{lcc}
\hline Items & RA & HD \\
\hline Number of patients & 8 & 6 \\
Sex & & \\
$\quad$ Men & 4 & 3 \\
$\quad$ Women & 4 & 3 \\
Age (years) & $45(39-49)$ & $43(37-48)$ \\
Disease duration (years) & $3.3(0.3-5.8)$ & $2.8(0.1-4.8)$ \\
CRP (mg/L) & $19.2(6-58)$ & - \\
ESR (mm/h) & $32(10-73)$ & - \\
\hline
\end{tabular}

CRP: C-reactive protein; ESR: erythrocyte sedimentation rate.

were 4828 differentially expressed genes in the RA SMSCs compared to the HD SMSCs. In addition, the mitogenactivated protein kinase (MAPK) signaling and rheumatoid arthritis pathways were both upregulated, and the p53 signaling pathway was downregulated. These findings could help explain the pathogenic mechanism of RA and potentially lead to RA SMSC-based therapeutic applications.

\section{Materials and Methods}

2.1. Cell Isolation and Culture. This study was approved by the ethics committee of Sun Yat-sen Memorial Hospital, and all study subjects provided written informed consent. Synovial tissue biopsies were obtained from 8 patients with $\mathrm{RA}$ and $6 \mathrm{HDs}$ using $3.5 \mathrm{~mm}$ grasping biopsy forceps under arthroscopy. The RA patients fulfilled the American College of Rheumatology revised criteria for rheumatoid arthritis [10], and patients who were undergoing arthroscopy for meniscus injury without any autoimmune disease or any signs of osteoarthritis were regarded as HDs for practical purposes. The clinical status of each subject is shown in Table 1. The synovial tissue samples were rinsed three times with phosphate-buffered saline (PBS; Biovision). The samples were then finely minced and digested with $0.2 \%$ collagenase type II (MP) in high-glucose Dulbecco's modified Eagle's medium (HDMEM; GIBCO) containing 10\% fetal bovine serum (FBS), $100 \mathrm{U} / \mathrm{mL}$ penicillin, and $100 \mathrm{U} / \mathrm{mL}$ streptomycin. After the samples were incubated overnight at $37^{\circ} \mathrm{C}$, cells were collected by centrifugation, rinsed three times with PBS, resuspended in HDMEM, plated in a T25 culture flask, and allowed to attach for 3 days. Nonadherent cells were removed by changing the medium, and the attached cells were cultured in HDMEM at $37^{\circ} \mathrm{C}$ in a humidified $5 \% \mathrm{CO}_{2}$ atmosphere until reaching confluence. The SMSCs were passaged upon reaching 80 90\% confluence as previously described [11], and cells from passages 3-5 were used for experiments.

2.2. Immunophenotypic Characterization of SMSCs. The immunophenotypic characterization of SMSCs was performed by flow cytometry. After SMSCs were digested with $0.25 \%$ trypsin, the cells were resuspended with PBS in flow cytometry tubes. Aliquots of $5.0 \times 10^{5}$ cells were incubated with conjugated monoclonal antibodies against CD34-PE,
CD44-FITC, CD45-FITC, CD90-PE, CD105-PE, and HLADR-PE or conjugated isotype controls (all from BD Pharmingen) for $30 \mathrm{~min}$ in the dark. Flow cytometry was performed using a Becton Dickinson FACSCalibur, and the data were analyzed by CellQuest software (Becton Dickinson).

2.3. Multipotent Differentiation of SMSCs. Three previously described procedures were used for the multipotent differentiation of SMSCs [11]. For osteogenic differentiation, SMSCs were cultured for 21 days in HDMEM containing $0.1 \mu \mathrm{M}$ dexamethasone (Sigma), $50 \mu \mathrm{M}$ vitamin C (Sigma), $10 \mathrm{mM} \beta$-glycerophosphate (Sigma), 10\% FBS, $100 \mathrm{U} / \mathrm{mL}$ penicillin, and $100 \mathrm{U} / \mathrm{mL}$ streptomycin and were then stained with alizarin red (Sigma). For chondrogenic differentiation, aliquots of $2.0 \times 10^{5}$ cells were centrifuged at $500 \times \mathrm{g}$ for $10 \mathrm{~min}$; then, the cells were cultured for 21 days in centrifuge tubes with chondrogenic culture medium, which contained $6.25 \mu \mathrm{g} / \mathrm{mL}$ insulin (Sigma), $6.25 \mu \mathrm{g} / \mathrm{mL}$ transferrin (Sigma), $6.25 \mu \mathrm{g} / \mathrm{mL}$ sodium selenite (Sigma), $1.25 \mu \mathrm{g} \mathrm{g} / \mathrm{mL}$ bovine serum albumin (BSA, Sigma), $1 \mathrm{mmol} / \mathrm{L}$ sodium pyruvate (Sigma), $37.5 \mu \mathrm{g} / \mathrm{mL}$ vitamin C (Sigma), $50 \mathrm{ng} / \mathrm{mL}$ transforming growth factor- $\beta_{1}$ (TGF- $\beta_{1}$, Sigma), $2.5 \%$ FBS, $100 \mathrm{U} / \mathrm{mL}$ penicillin, and $100 \mathrm{U} / \mathrm{mL}$ streptomycin. After these cells were harvested, they were stained with toluidine blue (Sigma). For adipogenic differentiation, SMSCs were cultured for 21 days in HDMEM containing $1 \mu \mathrm{M}$ dexamethasone, $200 \mu \mathrm{M}$ indomethacin, $0.5 \mathrm{mM} 3$-isobutyl-1methylxanthine, $10 \mu \mathrm{g} / \mathrm{mL}$ insulin, $10 \% \mathrm{FBS}, 100 \mathrm{U} / \mathrm{mL}$ penicillin, and $100 \mathrm{U} / \mathrm{mL}$ streptomycin and were then stained with oil red O (Sigma). All measurements were performed in triplicate. An inverted phase-contrast microscope (Nikon, Japan) was used to observe the cells and obtain images.

2.4. Total RNA Extraction and Quality Control. Total RNA was isolated from $8 \mathrm{RA}$ and $6 \mathrm{HD}$ SMSCs samples with $1 \mathrm{~mL}$ of TRIzol (Invitrogen) and was extracted using the phenol/chloroform method. Salt was washed away with $70 \%$ alcohol, and then total RNA was air-dried at room temperature. Total RNA was dissolved with an appropriate amount of RNase-free water. Standard denaturing agarose gel electrophoresis was used to assess RNA integrity, and a NanoDrop ND-100 was used to measure RNA quantity and quality.

2.5. RNA Labeling and Array Hybridization. Fourteen double-stranded cDNA (ds-cDNA) sequences were synthesized from the total RNA of 8 RA and 6 HD SMSC samples using an Invitrogen SuperScript ds-cDNA Synthesis kit. One $\mu \mathrm{g}$ of ds-cDNA was labeled in the Human $12 \times 135 \mathrm{~K}$ Gene Expression Array (Roche NimbleGen) with a NimbleGen One-Color DNA Labeling kit, according to the NimbleGen Gene Expression Analysis protocol (NimbleGen Systems, Inc.). Microarrays were hybridized with $4 \mu \mathrm{g}$ of Cy3-labeled ds-cDNA in NimbleGen Hybridization Buffer/Hybridization Component A in a hybridization chamber (Hybridization System-NimbleGen Systems, Inc.) at $42^{\circ} \mathrm{C}$ over 16 to $20 \mathrm{~h}$. After hybridization, the 14 microarrays were washed in an ozone-free environment using a NimbleGen Wash Buffer kit (NimbleGen Systems, Inc.). 
TABLE 2: Primers used for quantitative real-time polymerase chain reaction.

\begin{tabular}{lll}
\hline Gene & Forward primer & Reverse primer \\
\hline ARRB1 & AAAGGGACCCGAGTGTTCAAG & CGTCACATAGACTCTCCGCT \\
BDNF & GGCTTGACATCATTGGCTGAC & CATTGGGCCGAACTTTCTGGT \\
FGFR2 & AGCACCATACTGGACCAACAC & GGCAGCGAAACTTGACAGTG \\
PDGFRA & TGGCAGTACCCCATGTCTGAA & CCAAGACCGTCACAAAAAGGC \\
TGFBR1 & GCTGTATTGCAGACTTAGGACTG & TTTTTGTTCCCACTCTGTGGTT \\
CXCL12 & ATTCTCAACACTCCAAACTGTGC & ACTTTAGCTTCGGGTCAATGC \\
BL6 & ACTCACCTCTTCAGAACGAATTG & CCATCTTTGGAAGGTTCAGGTTG \\
CASP9 & GACCTCAACGCACAGTACGAG & AGGAGTCCCATGATGAGATTGT \\
\hline
\end{tabular}

2.6. Microarray Scanning and Data Analysis. The fourteen microarrays were scanned using an Axon GenePix 4000B microarray scanner (Molecular Devices Corporation) piloted by GenePix Pro 6.0 software (Axon). For grid alignment and expression data analysis, the scanned images were then imported into the NimbleScan software, and the expression data were normalized using the Robust Multichip Average algorithm and quantile normalization. The probe-level and gene-level files were generated after normalization, and all gene-level files were further analyzed according to the RA and HD groupings using Agilent GeneSpring GX software (version 12.0). A scatter plot was used to visualize the data and access variation between RA and HD SMSCs. Volcano plot filtering was used to identify the genes differentially expressed between the two groups, and genes that exhibited greater than or equal to 2.0-fold change were selected for data analysis. Hierarchical clustering was also performed using Agilent GeneSpring GX software, and the variability between RA patient samples and HD samples was high while that in RA patient samples and HD samples was very low, which means that all these chips were available for further analysis. Then, gene symbols were uploaded to the Gene Ontology (GO, http://www.geneontology.org/) for GO annotation and enrichment analysis. The differentially expressed genes were used to perform pathway analyses in the most recent Kyoto Encyclopedia of Genes and Genomes (KEGG, http://www.genome.jp/kegg/) database.

2.7. Quantitative Real-Time Polymerase Chain Reaction ( $q R T$ PCR). Total RNA of the SMSCs was extracted and transcribed into cDNA as described. A LightCycler 480 RealTime PCR System (Roche, Basel) was used to perform the qRT-PCR analyses with a SYBR Premix Ex Taq II kit (Takara, Otsu). The PCR primers are listed in Table 2. PCR amplification consisted of $95^{\circ} \mathrm{C}$ for $30 \mathrm{~s}$, followed by 40 cycles of $95^{\circ} \mathrm{C}$ for $5 \mathrm{~s}$ and $60^{\circ} \mathrm{C}$ for $20 \mathrm{~s}$. Specificity was verified by a melting curve analysis, and the relative amounts of target gene mRNA were normalized to the expression of the housekeeping gene GAPDH.

2.8. Western Blot. After being washed with ice-cold PBS three times, RA and HD SMSCs were lysed in $0.06 \mathrm{~mL}$ of cell lysis buffer (Beyotime) supplemented with a cocktail of protease inhibitors on ice for $30 \mathrm{~min}$. The cells were centrifuged at $14,000 \times \mathrm{g}$ for $30 \mathrm{~min}$ at $4^{\circ} \mathrm{C}$, and then supernatants were collected. A BCA Protein Assay kit (CWBiotech) was used to measure the protein concentrations. After all the samples were boiled with 20\% sample loading buffer (Beyotime), $20 \mu \mathrm{L}$ of each protein extract was electrophoresed using $10 \%$ sodium dodecylsulfate-polyacrylamide gel electrophoresis and then transferred to a polyvinylidene fluoride (PVDF) membrane (Millipore). The PVDF membranes were blocked in Tris-buffered saline with Tween-20 (Cell Signaling Technology) and 5\% nonfat milk for $60 \mathrm{~min}$ at room temperature and then incubated overnight at $4^{\circ} \mathrm{C}$ with primary antibodies against GAPDH, p53, p-JNK, p-ERK, and p-p38 (dilution 1:1000; Cell Signaling Technology). The PVDF membranes were incubated with appropriate secondary antibodies (dilution 1:3000; Santa Cruz) for $60 \mathrm{~min}$ at room temperature. Then, protein expression levels were detected using enhanced chemiluminescence (Millipore) and quantified using ImageJ software (National Institutes of Health, USA).

2.9. Statistical Analysis. The results were analyzed using GraphPad Prism 6 (GraphPad Software, Inc.) and presented as the mean \pm standard deviation (SD). Student's $t$-test was used to compare means between two groups. $P$ values $<0.05$ were considered statistically significant.

\section{Results}

3.1. Identification of SMSCs via Immunophenotype and Multipotent Differentiation In Vitro. The immunophenotypic identification of SMSCs in the RA and HD groups was performed by flow cytometry. The cells in both groups were positive for CD44, CD90, and CD105 and negative for CD34, CD45, and HLA-DR, and no significant differences were found $(P$ value $>0.05$, Figure 1$)$.

After induction for $21 \mathrm{~d}$, the SMSCs had clearly differentiated into osteocytes, chondrocytes, and adipocytes. Osteogenesis was indicated by mineralization nodes that were observed after being stained with alizarin red (Figure 2(a)); chondrogenesis was indicated by glycosaminoglycans that were stained with toluidine blue (Figure 2(b)); and adipogenesis was indicated by lipid vacuoles that were stained with oil red $\mathrm{O}$ (Figure 2(c)). Together with the immunophenotypic 


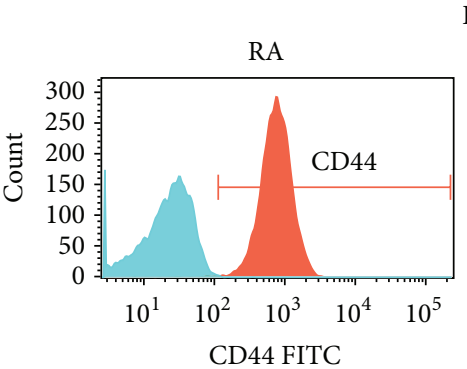

Positive
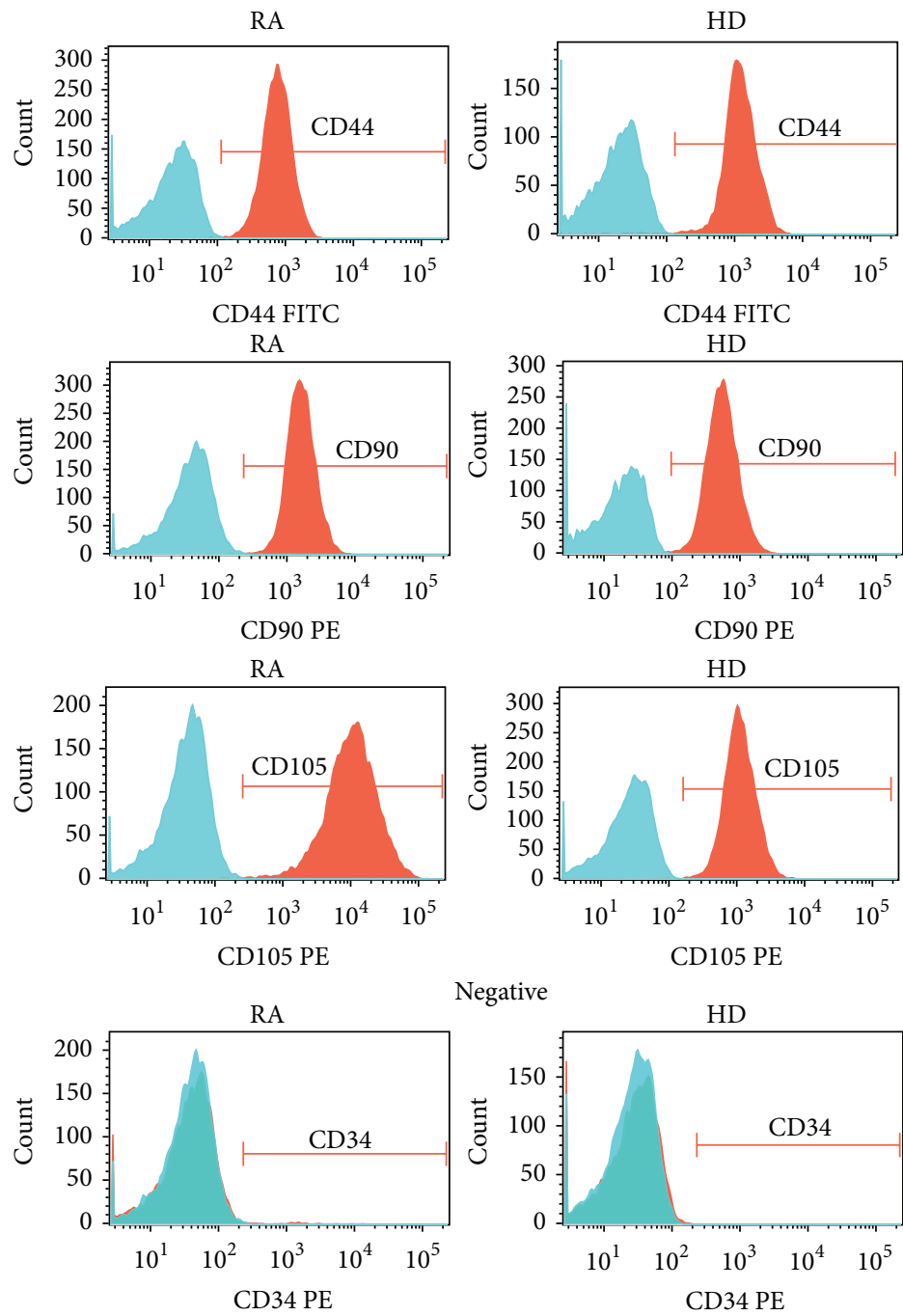

Negative
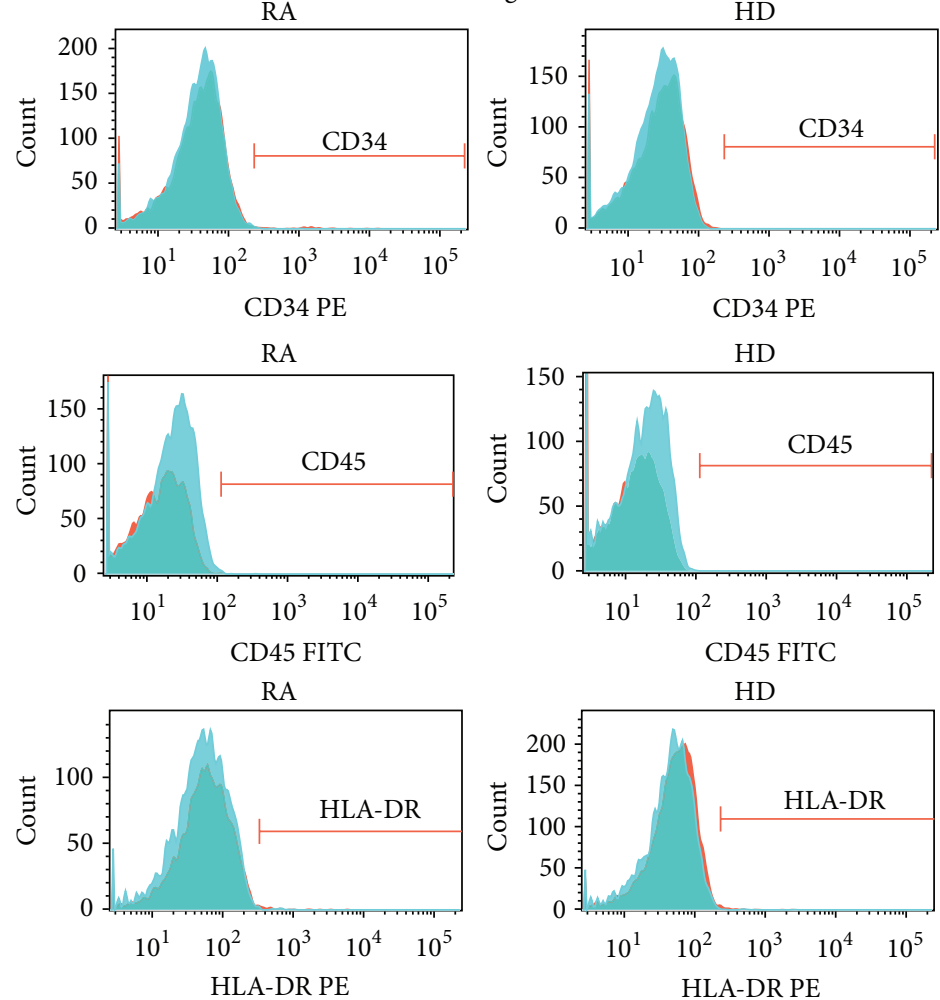

Figure 1: SMSC immunophenotypes. SMSCs from RA patients and HDs were positive for CD44, CD90, and CD105 and negative for CD34, CD45, and HLA-DR. Blue areas indicate background fluorescence of isotype control IgG, and red areas represent the fluorescence of the corresponding immunophenotype. The $x$-axis represents fluorescence intensity, and the $y$-axis represents cell count. 
RA

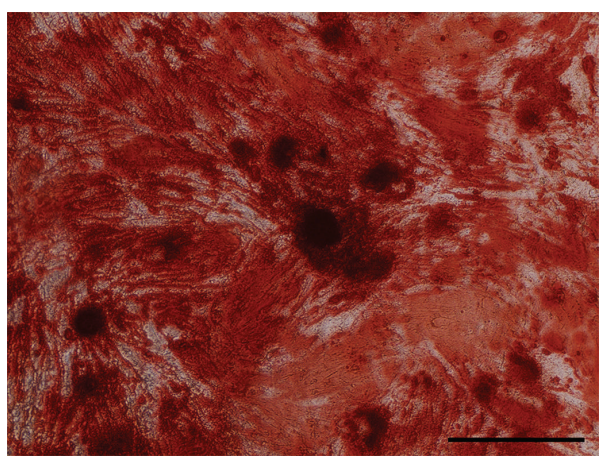

(a)

RA

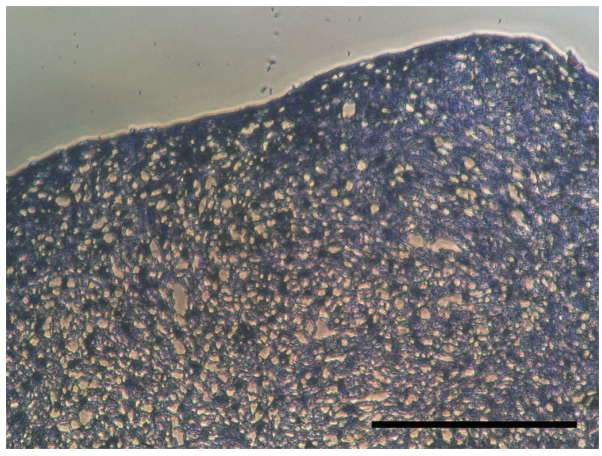

(b)

RA

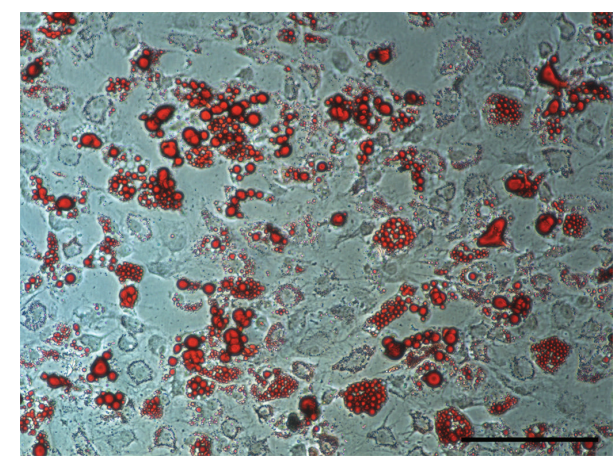

HD

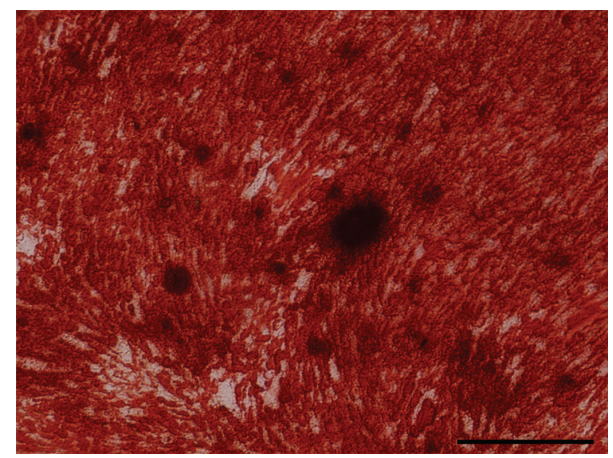

HD

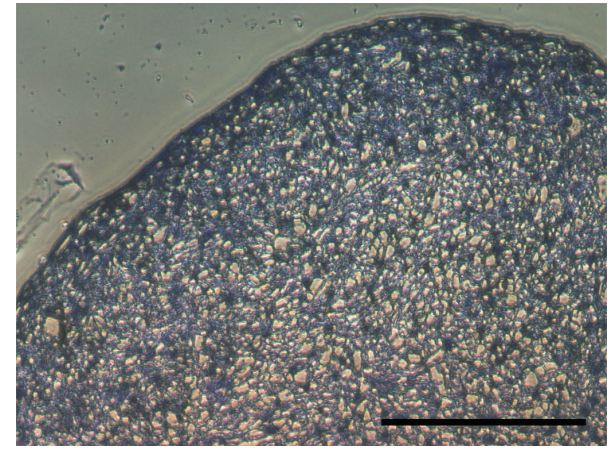

HD

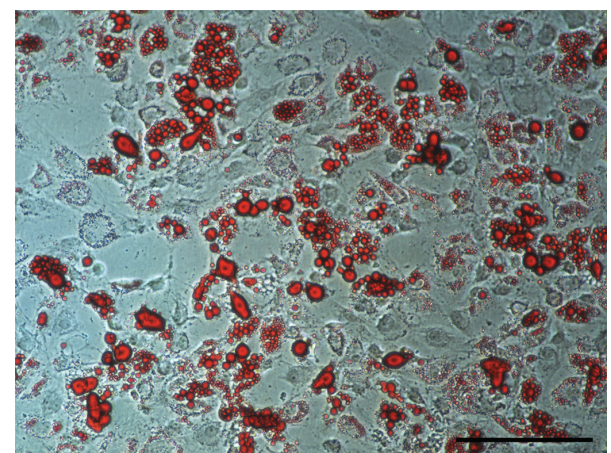

(c)

FIGURE 2: Triple SMSC differentiations. Osteogenic, chondrogenic, and adipogenic differentiation of SMSCs from RA patients and HDs were observed using an inverted phase-contrast microscope. (a) Osteogenic differentiation was indicated by mineralization nodes stained with alizarin red (scale bar: $200 \mu \mathrm{m}$ ). (b) Chondrogenic differentiation was identified by glycosaminoglycans stained with toluidine blue (scale bar: $200 \mu \mathrm{m}$ ). (c) Adipogenic differentiation was identified by lipid vacuoles stained with oil red O (scale bar: $200 \mu \mathrm{m}$ ).

identifications mentioned above, these results indicated that SMSCs from the RA and HD groups fulfilled the defining criteria for MSCs [12].

3.2. Scatter Plot and Volcano Plots. All the data are shown in a scatter plot, which is a useful method for visualizing and assessing interchip variation (Figure 3(a)). Differential expression between RA and HD SMSCs was visualized using volcano plots (Figure 3(b)), which are constructed using foldchange values and $P$ values and allow the visualization of the relationship between fold-change, that is, the magnitude of change, and statistical significance, which encompasses both the magnitude of change and variability.

3.3. GO Analysis. There were 4828 genes that were differentially expressed between the RA and HD groups $(P<0.05)$, of which 3117 were upregulated and 1711 were downregulated. GO was used to analyze the differentially expressed genes in three domains, that is, the biological process, cellular component, and molecular function domains. Counts of 


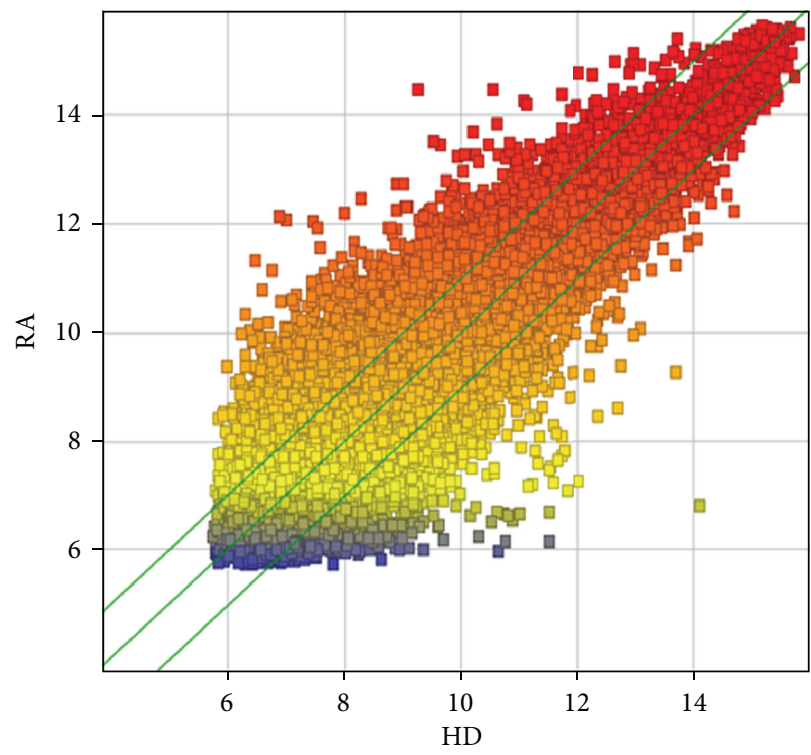

(a)

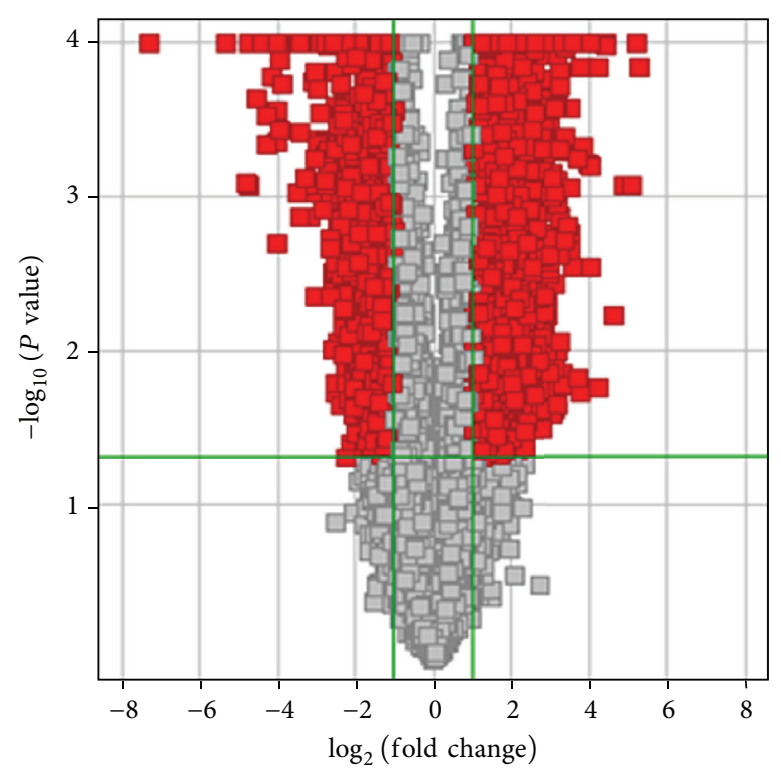

(b)

FIGURE 3: Scatter plot and volcano plots of differentially expressed genes. (a) Differentially expressed genes of SMSCs from both RA patients and HDs are shown in the scatter plot; each value represents a normalized signal value from each gene $\left(\log _{2}\right.$ scale). Fold-change lines are shown as green lines in the scatter plot, and gene fold-changes $\geq 2.0$ between the RA and HD groups are shown above the top green line and below the bottom green line. (b) The volcano plots were constructed using fold-change values $\left(\log _{2}\right.$ scale) and $P$ values (- $\log _{10}$ scale). The vertical lines correspond to 2.0 -fold change up and down and the horizontal line represents a $P$ value of 0.05 . The red point in the plot represents the significantly differentially expressed genes.

the top ten significantly enriched terms of the upregulated and downregulated genes are shown in Figure 4.

In the biological process domain, there were 797 significant functional description nodes in the upregulated genes and 380 significant functional description nodes in the downregulated genes. The top ten significantly enriched terms in the upregulated genes were cellular process, metabolic process, primary metabolic process, cellular metabolic process, biological regulation, regulation of biological process, regulation of cellular process, macromolecule metabolic process, cellular macromolecule metabolic process, and response to stimulus. The top ten significantly enriched terms in the downregulated genes were cellular process, biological regulation, regulation of biological process, response to stimulus, multicellular organismal process, cellular response to stimulus, signaling, cellular component organization or biogenesis, development process, and cellular component organization (Table 3 ).

In the cellular component domain, there were 125 significant functional description nodes in the upregulated genes and 52 significant functional description nodes in the downregulated genes. The top ten significantly enriched terms in the upregulated genes were cell part, cell, intracellular, intracellular part, intracellular organelle, organelle, cytoplasm, intracellular membrane-bounded organelle, membrane-bounded organelle, and cytoplasmic part. The top ten significantly enriched terms in the downregulated genes were macromolecular complex, non-membranebounded organelle, intracellular non-membrane-bounded organelle, protein complex, cytoskeleton, plasma membrane part, cytosol, cytoskeletal part, integral to plasma membrane, and intrinsic to plasma membrane (Table 4).

In the molecular function domain, there were 163 significant functional description nodes in the upregulated genes and 44 significant functional description nodes in the downregulated genes. The top ten significantly enriched terms in the upregulated genes were binding, protein binding, catalytic activity, ion binding, cation binding, metal ion binding, hydrolase activity, nucleotide binding, purine nucleotide binding, and ribonucleotide binding. The top ten significantly enriched terms in the downregulated genes were binding, protein binding, identical protein binding, sequence-specific DNA binding, structural molecule activity, cytokine binding, growth factor activity, protein C-terminus binding, growth factor binding, and tubulin binding (Table 5).

3.4. Screening of Signaling Pathways Related to RA SMSCs. Based on the most recent KEGG database, we carried out KEGG pathway analyses to determine the pivotal signaling pathways of the differentially expressed genes. The two highest enrichment scores for the upregulated genes in the RA group were hsa04010 (MAPK signaling pathway) and hsa05323 (rheumatoid arthritis pathway). Moreover, the highest enrichment score for the downregulated genes in the RA group was hsa04115 (p53 signaling pathway) (Table 5). In the MAPK signaling pathway, there were 47 differentially expressed genes: ARRB1, ATF2, BDNF, CACNA1C, CASP3, CHUK, CRK, DUSP10, DUSP3, DUSP5, EGFR, FGF10, FGF7, FGFR1, FGFR2, GNG12, HSPA2, HSPA6, HSPA8, IL1R1, JUN, MAP2K4, MAP3K12, MAP3K2, MAP3K4, MAP3K5, 


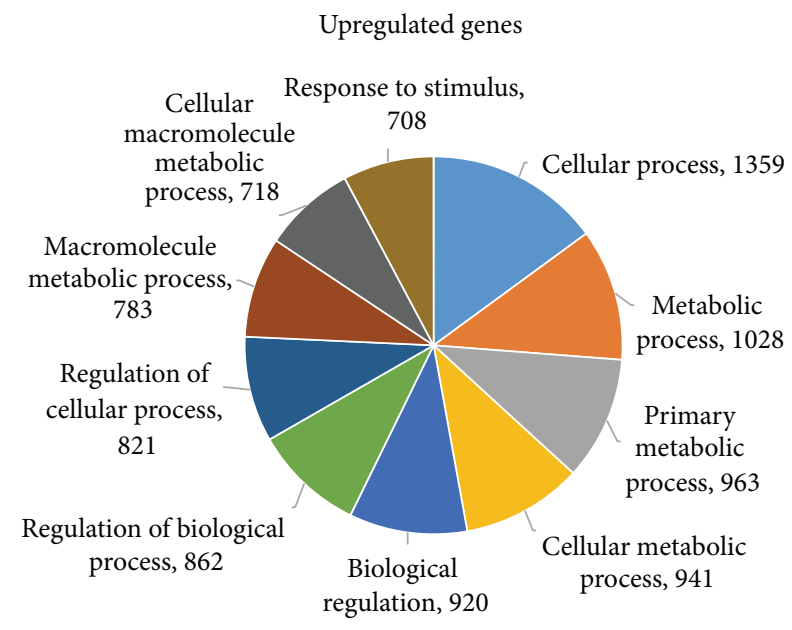

(a)

\section{Upregulated genes}

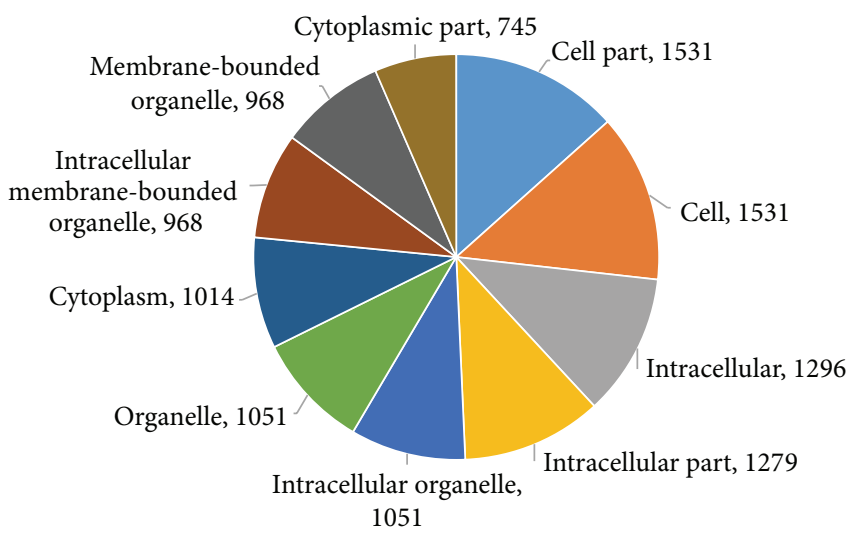

(b)
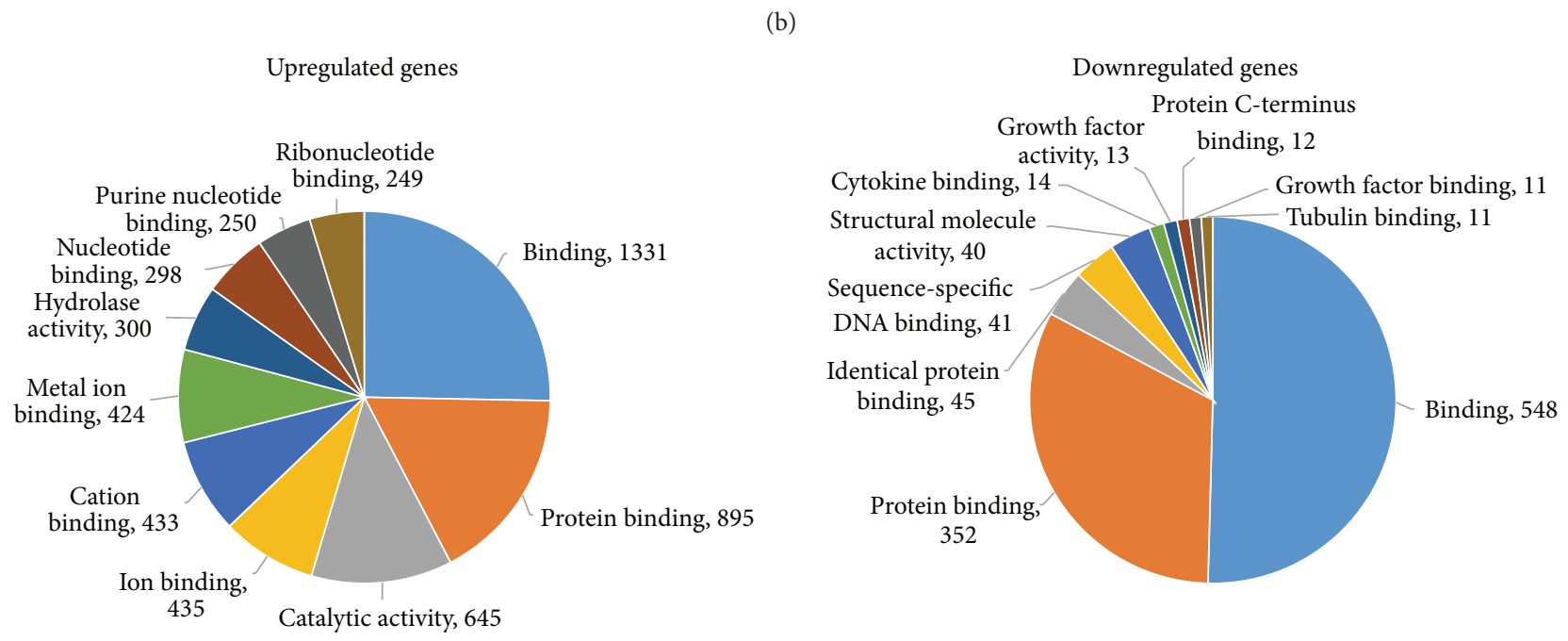

(c)
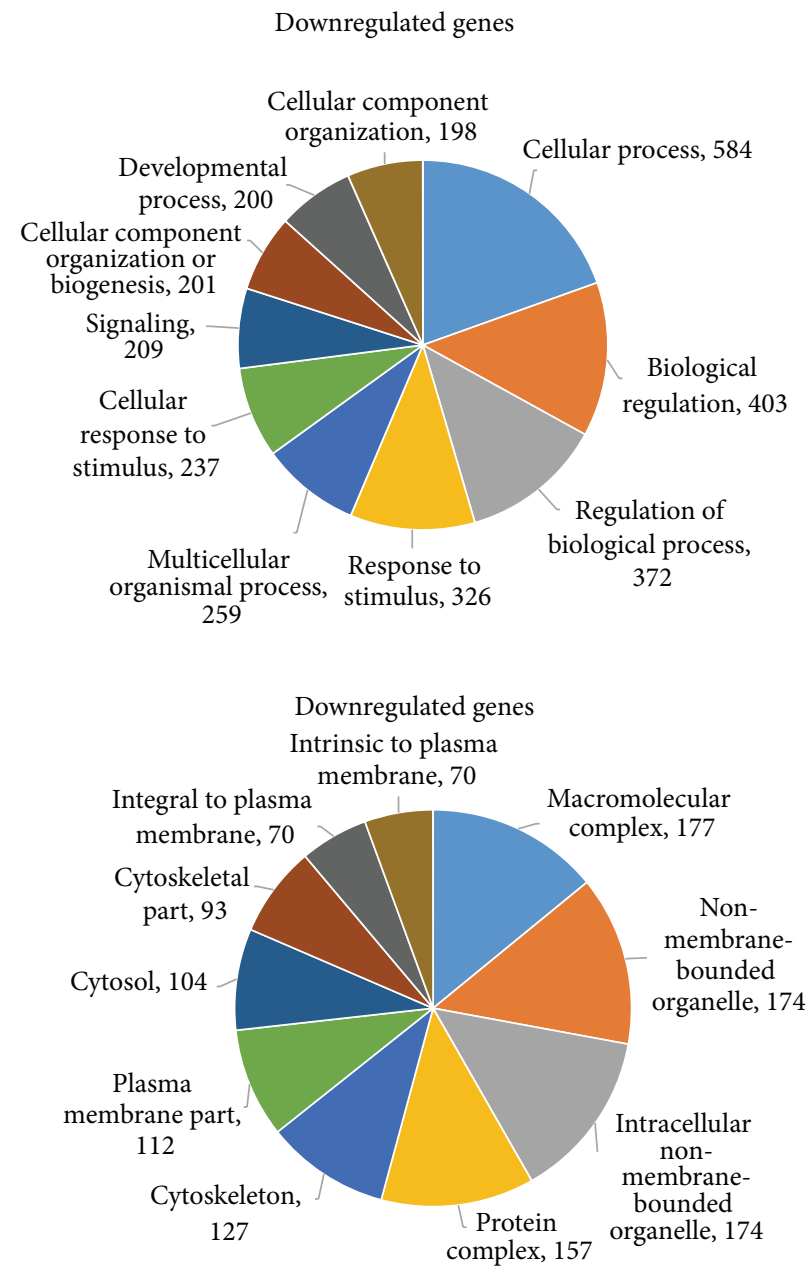

Figure 4: Gene Ontology (GO) analysis results of the genes differentially expressed in SMSCs from RA patients and HDs. Counts of the top ten significantly enriched terms of upregulated and downregulated genes in the biological process, cellular component, and molecular function domains are shown in (a), (b), and (c), respectively. 
TABLE 3: GO analysis of RA SMSCs with different biological processes related to differentially expressed genes.

\begin{tabular}{|c|c|c|c|c|c|c|}
\hline GO ID & Term & Regulation & Number of genes & $\begin{array}{l}\text { Percentage of } \\
\text { total gene } \\
\text { number }\end{array}$ & Fold enrichment & $P$ value \\
\hline GO:0009987 & Cellular process & Up & 1359 & $43.60 \%$ & 1.078949506 & $3.45058 E-16$ \\
\hline GO:0008152 & Metabolic process & Up & 1028 & $32.98 \%$ & 1.127180781 & $2.26411 E-11$ \\
\hline GO:0044238 & Primary metabolic process & up & 963 & $30.90 \%$ & 1.163226478 & $3.42172 E-14$ \\
\hline GO:0044237 & Cellular metabolic process & Up & 941 & $30.19 \%$ & 1.157274694 & $8.30096 E-13$ \\
\hline GO:0065007 & Biological regulation & Up & 920 & $29.52 \%$ & 1.134286715 & $9.96280 E-10$ \\
\hline GO:0050789 & Regulation of biological process & Up & 862 & $27.65 \%$ & 1.130113501 & $2.91311 E-08$ \\
\hline GO:0050794 & Regulation of cellular process & Up & 821 & $26.34 \%$ & 1.133035445 & $7.34418 E-08$ \\
\hline GO:0043170 & $\begin{array}{l}\text { Cellular macromolecule } \\
\text { metabolic process }\end{array}$ & Up & 718 & $23.03 \%$ & 1.162237783 & $1.77085 E-08$ \\
\hline GO:0050896 & Response to stimulus & Up & 708 & $22.71 \%$ & 1.108798503 & $7.11907 E-05$ \\
\hline GO:0006807 & $\begin{array}{l}\text { Nitrogen compound metabolic } \\
\text { process }\end{array}$ & Up & 568 & $18.22 \%$ & 1.063792239 & 0.028072195 \\
\hline GO:0009987 & Cellular process & Down & 584 & $34.13 \%$ & 1.030034922 & 0.031637828 \\
\hline GO:0065007 & Biological regulation & Down & 403 & $23.55 \%$ & 1.103818079 & 0.001406004 \\
\hline GO:0050789 & Regulation of biological process & Down & 372 & $21.74 \%$ & 1.083465722 & 0.012748648 \\
\hline GO:0050896 & Response to stimulus & Down & 326 & $19.05 \%$ & 1.134212455 & 0.001202522 \\
\hline GO:0032501 & Multicellular organismal process & Down & 259 & $15.14 \%$ & 1.094089824 & 0.036294594 \\
\hline GO:0051716 & Cellular response to stimulus & Down & 237 & $13.85 \%$ & 1.208258943 & 0.000275304 \\
\hline GO:0023052 & Signaling & Down & 209 & $12.22 \%$ & 1.142323863 & 0.012379382 \\
\hline GO:0071840 & $\begin{array}{l}\text { Cellular component organization } \\
\text { or biogenesis }\end{array}$ & Down & 201 & $11.75 \%$ & 1.275102033 & $4.79255 E-05$ \\
\hline GO:0032502 & Developmental process & Down & 200 & $11.69 \%$ & 1.192861477 & 0.002106076 \\
\hline GO:0016043 & Cellular component organization & Down & 198 & $11.57 \%$ & 1.295981777 & $2.02658 E-05$ \\
\hline
\end{tabular}

TABLE 4: GO analysis of RA SMSCs with different cellular components related to differentially expressed genes.

\begin{tabular}{|c|c|c|c|c|c|c|}
\hline GO ID & Term & Regulation & Number of genes & $\begin{array}{c}\text { Percentage of } \\
\text { total gene } \\
\text { number }\end{array}$ & Fold enrichment & $P$ value \\
\hline GO:0005623 & Cell & Up & 1531 & $49.12 \%$ & 1.027156657 & $2.40220 E-05$ \\
\hline GO:0005622 & Intracellular & Up & 1296 & $41.58 \%$ & 1.143609988 & $4.14799 E-22$ \\
\hline GO:0043229 & Intracellular organelle & Up & 1051 & $33.72 \%$ & 1.120831397 & $8.22135 E-10$ \\
\hline GO:0005737 & Cytoplasm & Up & 1014 & $32.53 \%$ & 1.277346130 & $3.84962 E-31$ \\
\hline GO:0043231 & $\begin{array}{l}\text { Intracellular } \\
\text { membrane-bounded } \\
\text { organelle }\end{array}$ & Up & 968 & $31.06 \%$ & 1.147230634 & $3.86604 E-11$ \\
\hline GO:0044444 & Cytoplasmic part & Up & 745 & $23.90 \%$ & 1.315817744 & $3.25995 E-22$ \\
\hline GO:0044446 & Intracellular organelle part & Up & 607 & $19.47 \%$ & 1.163042085 & $1.40257 E-06$ \\
\hline GO:0005634 & Nucleus & Up & 565 & $18.13 \%$ & 1.073142161 & 0.017245693 \\
\hline GO:0032991 & Macromolecular complex & Up & 361 & $11.58 \%$ & 1.081921665 & 0.042183953 \\
\hline GO:0043234 & Protein complex & Up & 314 & $10.07 \%$ & 1.127231548 & 0.008327837 \\
\hline GO:0032991 & Macromolecular complex & Down & 177 & $10.34 \%$ & 1.18550075 & 0.006000202 \\
\hline GO:0043232 & $\begin{array}{l}\text { Intracellular } \\
\text { non-membrane-bounded } \\
\text { organelle }\end{array}$ & Down & 174 & $10.17 \%$ & 1.521121372 & $2.70923 E-09$ \\
\hline GO:0043234 & Protein complex & Down & 157 & $9.18 \%$ & 1.259572268 & 0.000893262 \\
\hline GO:0005856 & Cytoskeleton & Down & 127 & $7.42 \%$ & 1.939965930 & $1.27657 E-13$ \\
\hline GO:0044459 & Plasma membrane part & Down & 112 & $6.55 \%$ & 1.210497238 & 0.017123710 \\
\hline GO:0005829 & Cytosol & Down & 104 & $6.08 \%$ & 1.201689499 & 0.025595975 \\
\hline GO:0005887 & $\begin{array}{l}\text { Integral to plasma } \\
\text { membrane }\end{array}$ & Down & 70 & $4.09 \%$ & 1.334366755 & 0.007950984 \\
\hline GO:0031226 & $\begin{array}{l}\text { Intrinsic to plasma } \\
\text { membrane }\end{array}$ & Down & 70 & $4.09 \%$ & 1.309313339 & 0.011994087 \\
\hline GO:0015630 & Microtubule cytoskeleton & Down & 61 & $3.57 \%$ & 2.133880899 & $1.80251 E-08$ \\
\hline GO:0005694 & Chromosome & Down & 41 & $2.40 \%$ & 1.75267224 & 0.000358288 \\
\hline
\end{tabular}


TABLE 5: GO analysis of RA SMSCs with different molecular functions related to differentially expressed genes.

\begin{tabular}{|c|c|c|c|c|c|c|}
\hline GO ID & Term & Regulation & Number of genes & $\begin{array}{c}\text { Percentage of } \\
\text { total gene } \\
\text { number }\end{array}$ & Fold enrichment & $P$ value \\
\hline GO:0005488 & Binding & Up & 1331 & $42.70 \%$ & 1.107406186 & $6.17161 E-19$ \\
\hline GO:0005515 & Protein binding & Up & 895 & $28.71 \%$ & 1.259276446 & $1.94840 E-23$ \\
\hline GO:0003824 & Catalytic activity & Up & 645 & $20.69 \%$ & 1.218132345 & $6.46805 E-11$ \\
\hline GO:0043167 & Ion binding & Up & 435 & $13.96 \%$ & 1.110490713 & 0.004421943 \\
\hline GO:0043169 & Cation binding & Up & 433 & $13.89 \%$ & 1.108515605 & 0.005140801 \\
\hline GO:0046872 & Metal ion binding & Up & 424 & $13.60 \%$ & 1.097341066 & 0.011174111 \\
\hline GO:0016787 & Hydrolase activity & Up & 300 & $9.62 \%$ & 1.291345373 & $4.92602 E-07$ \\
\hline GO:0000166 & Nucleotide binding & Up & 298 & $9.56 \%$ & 1.314022658 & $1.04955 E-07$ \\
\hline GO:0017076 & Purine nucleotide binding & Up & 250 & $8.02 \%$ & 1.329326120 & $5.67347 E-07$ \\
\hline GO:0032553 & Ribonucleotide binding & Up & 249 & $7.99 \%$ & 1.332560002 & $4.92004 E-07$ \\
\hline GO:0005488 & Binding & Down & 548 & $32.03 \%$ & 1.037520988 & 0.031108598 \\
\hline GO:0005515 & Protein binding & Down & 352 & $20.57 \%$ & 1.127010990 & 0.001007060 \\
\hline GO:0042802 & Identical protein binding & Down & 45 & $2.63 \%$ & 1.385306122 & 0.016715118 \\
\hline GO:0043565 & Sequence-specific DNA binding & Down & 41 & $2.40 \%$ & 1.388762475 & 0.020934665 \\
\hline GO:0005198 & Structural molecule activity & Down & 40 & $2.34 \%$ & 1.491048874 & 0.007638802 \\
\hline GO:0019955 & Cytokine binding & Down & 14 & $0.82 \%$ & 2.778713450 & 0.000496933 \\
\hline GO:0008083 & Growth factor activity & Down & 13 & $0.76 \%$ & 1.793577236 & 0.030091084 \\
\hline GO:0008022 & Protein C-terminus binding & Down & 12 & $0.70 \%$ & 1.810133333 & 0.034106543 \\
\hline GO:0019838 & Growth factor binding & Down & 11 & $0.64 \%$ & 2.262666667 & 0.009349359 \\
\hline GO:0015631 & Tubulin binding & Down & 11 & $0.64 \%$ & 2.183274854 & 0.012057826 \\
\hline
\end{tabular}

TABLE 6: KEGG pathway analysis results for RA SMSCs.

\begin{tabular}{|c|c|c|c|c|c|}
\hline Pathway ID & Definition & Regulation & Selection counts & Gene names & $P$ value \\
\hline hsa04010 & MAPK signaling pathway & Up & 47 & $\begin{array}{l}\text { ARRB1, ATF2, BDNF, CACNA1C, CASP3, CHUK, } \\
\text { CRK, DUSP10, DUSP3, DUSP5, EGFR, FGF10, FGF7, } \\
\text { FGFR1, FGFR2, GNG12, HSPA2, HSPA6, HSPA8, } \\
\text { IL1R1, JUN, MAP2K4, MAP3K12, MAP3K2, MAP3K4, } \\
\text { MAP3K5, MAP3K7, MAP3K8, MAP4K3, MAP4K4, } \\
\text { MAPK10, MAX, NF1, PDGFRA, PLA2G4A, PPM1A, } \\
\text { PPP3CB, RAPGEF2, RPS6KA3, RRAS2, SOS1, SOS2, } \\
\text { STK3, TGFB3, TGFBR1, TGFBR2, and ZAK }\end{array}$ & 0.002378379 \\
\hline hsa05323 & Rheumatoid arthritis & Up & 18 & $\begin{array}{l}\text { ANGPT1, ATP6V0A1, ATP6V1A, ATP6V1C2, } \\
\text { ATP6V1G1, CCL2, CTSK, CXCL1, CXCL12, CXCL5, } \\
\text { CXCL6, HLA-DQA1, ICAM1, IL6, JUN, MMP1, } \\
\text { MMP3, and TGFB3 }\end{array}$ & 0.018097090 \\
\hline hsa04115 & p53 signaling pathway & Down & 13 & $\begin{array}{l}\text { BBC3, CASP9, CCNB1, CCNB2, GADD45B, GTSE1, } \\
\text { IGF1, IGFBP3, RPRM, RRM2, SERPINE1, SESN2, and } \\
\text { SESN3 }\end{array}$ & 0.000017539 \\
\hline
\end{tabular}

MAP3K7, MAP3K8, MAP4K3, MAP4K4, MAPK10, MAX, NF1, PDGFRA, PLA2G4A, PPM1A, PPP3CB, RAPGEF2, RPS6KA3, RRAS2, SOS1, SOS2, STK3, TGFB3, TGFBR1, TGFBR2, and ZAK (Table 6). In the rheumatoid arthritis pathway, 18 genes were expressed differentially, including ANGPT1, ATP6V0A1, ATP6V1A, ATP6V1C2, ATP6V1G1, CCL2, CTSK, CXCL1, CXCL12, CXCL5, CXCL6, HLADQA1, ICAM1, IL6, JUN, MMP1, MMP3, and TGFB3. In the p53 signaling pathway, there were 13 differentially expressed genes: BBC3, CASP9, CCNB1, CCNB2, GADD45B,
GTSE1, IGF1, IGFBP3, RPRM, RRM2, SERPINE1, SESN2, and SESN3. The expression levels of several genes mentioned above were verified by qRT-PCR, including ARRB1, BDNF, FGFR2, PDGFRA, TGFBR1, CXCL12, IL6, BBC3, and CASP9 (Figure 5). Moreover, to further investigate the expression of p53 and MAPK signaling pathways, the expression levels of p53, p-JNK, p-ERK, and p-p38 were detected via western blot (Figure 6). Interestingly, the level of p53 expression was significantly lower and the levels of p-JNK, p-ERK, and pP38 expression were significantly higher in RA SMSCs than 


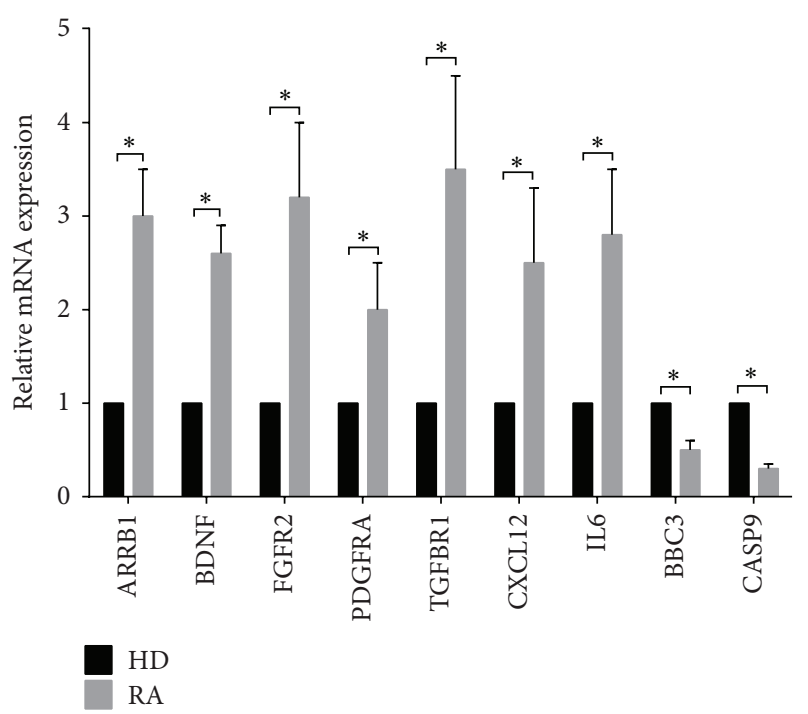

FIGURE 5: Quantitative real-time polymerase chain reaction (qRTPCR) results of genes differentially expressed in SMSCs from RA patients and HDs. qRT-PCR was used to verify partial differentially expressed genes in the MAPK signaling, rheumatoid arthritis, and p53 pathways, including ARRB1, BDNF, FGR2, PDGFRA, TGFBR1, CXCL12, IL6, BBC3, and CASP9. The internal marker gene GAPDH was used for normalization. ${ }^{*} P<0.05$.

in HD SMSCs. These findings further suggested that the MAPK pathway was upregulated and the p53 pathway was downregulated in RA SMSCs compared with HD SMSCs.

\section{Discussion}

In our previous work, we discovered that MSCs from patients with ankylosing spondylitis showed dysfunctional immunomodulation and differentiation, which may play an important role in the pathogenesis of ankylosing spondylitis or other chronic autoimmune diseases [13-16]. However, the immunomodulatory properties and differentiation capacities of RA SMSCs remain largely unknown. More than half of the risk of developing RA is due to genetic factors [17]. Nevertheless, except the PTPN22 and HLA genes, no major pathogenic genes have yet been identified to be associated with RA [1]. In this study, we explored the differences in whole-genome expression and signaling pathways of SMSCs from RA patients and HDs, which may lead to the discovery of the pathogenesis of RA. Our work identified 4828 differentially expressed genes in the RA group compared to the HD group, of which 3117 genes were upregulated and 1711 genes were downregulated.

The GO analysis showed that the genes with significant differences in the biological process domain were mostly related to cellular processes; in particular, upregulated genes were related to metabolic processes, and downregulated genes were related to biological regulation. In the cellular component domain, the upregulated genes were mainly related to intracellular organelles, particularly to the membrane-bounded organelles. Interestingly, intracellular non-membrane-bounded organelles accounted for the second largest proportion in downregulated genes. For the molecular function domain, the upregulated genes were mainly related to binding, including protein binding, ion binding, cation binding, metal ion binding, nucleotide binding, purine nucleotide binding, and ribonucleotide binding and catalytic activity, for example, hydrolase activity. Moreover, the downregulated genes were also mainly related to binding, especially protein binding.

The KEGG pathway analysis results showed that the MAPK signaling, rheumatoid arthritis, and p53 signaling pathways were differentially expressed in RA and HD SMSCs. The MAPK signaling pathway not only involves cytokine production and RA pathogenesis [18-20] but also regulates MSC differentiation, immunoregulation, and apoptosis [2124], indicating that the MAPK signaling pathway may play an important role in RA SMSCs. We found 47 differentially expressed genes in the MAPK signaling pathway, some of which are involved in the immune system, inflammatory diseases, or MSC biology. For instance, high ARRB1 expression is related to the pathogenesis of experimental autoimmune encephalomyelitis and multiple sclerosis [25]. In addition, brain-derived neurotrophic factor (BDNF) has a neuroprotective role in experimental autoimmune encephalomyelitis and multiple sclerosis, which may have important implications for the corresponding therapies [26, 27]. Interestingly, $\mathrm{BDNF}$ expression has been reported to be higher in RA synovial tissue [28], and our results showed that BDNF expression was also higher in RA SMSCs, suggesting that BDNF expression in RA SMSCs is involved in the pathogenesis of RA. FGF-2 and Anosmin-1, which are markers for the level of inflammation of multiple sclerosis lesions, participate in oligodendrocyte precursor cell migration via FGF receptor 1 (FGFR1) [29]. Intrarenal mRNA levels of PDGFRA are significantly enriched in patients with lupus nephritis [30]. TGFBR1 can control the immunomodulatory properties of MSCs via regulating their IL6 and indoleamine 2,3-dioxygenase secretions [31]. In addition, FGFR1 [32], FGFR2 [33], CACNA1C [34], GNG12 [35], MAP3K8 [36], and Nf1 [37] are involved in the differentiation potential of MSCs, indicating that some differences in the differentiation capacities of RA and HD SMSCs might exist. Our results showed that the genes upregulated in RA SMSCs related to the MAPK signaling pathway may be broadly involved in both the pathogenesis of RA and the biology of RA SMSCs; however, further experiments should be conducted to explore the functions of these genes in RA SMSCs.

The JNK pathway, the ERK pathway, and the p38 pathway are three well-defined MAPK pathways, and the phosphorylation of JNK, ERK, and p38 can result in the catalytic activation of MAPK signaling [38]. The levels of p-JNK, p-ERK, and p-p38 expression were significantly higher in RA SMSCs than in HD SMSCs, further suggesting that the MAPK pathway was upregulated in RA SMSCs compared with HD SMSCs.

The rheumatoid arthritis pathway was also upregulated in RA SMSCs, with 18 differentially expressed genes. Proinflammatory cytokine-related genes, such as CCL2, CXCL1, 

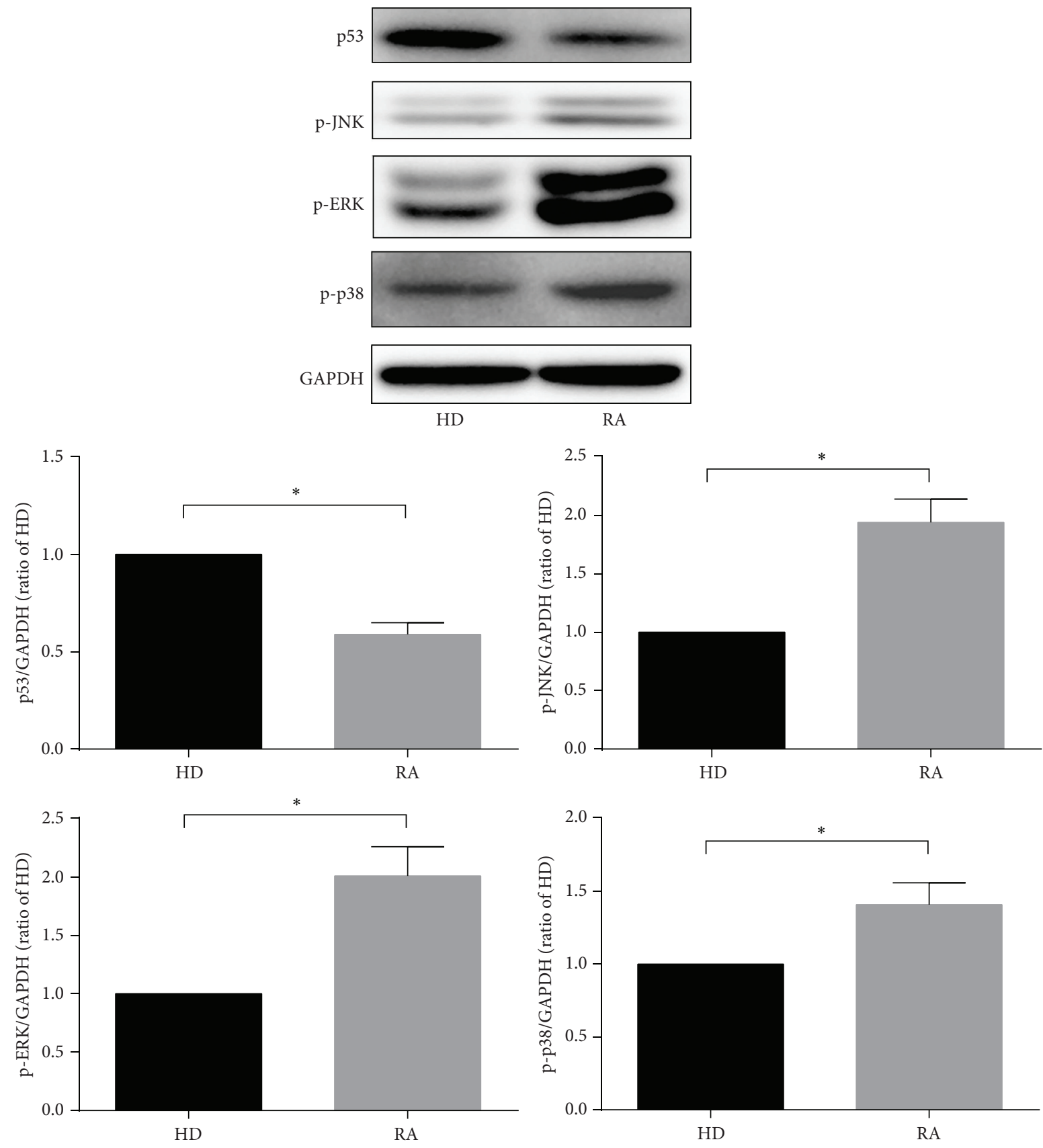

FIGURE 6: Western blot results of p53, p-JNK, p-ERK, and p-p38 expressed in SMSCs from RA patients and HDs. Western blot was used to determine the levels of $\mathrm{p} 53$ expression in the p53 pathway and p-JNK, p-ERK, and p-p38 expression in the MAPK signaling pathway. The internal marker protein GAPDH was used for normalization. ${ }^{*} P<0.05$.

CXCL5, CXCL6, CXCL12, and IL6, were upregulated in RA SMSCs. After stimulating CD4+ T cells and monocytes with CXCL12, osteoclast differentiation and $\mathrm{T}$ cell activation were enhanced via elevated expression levels of RANKL and TNF$\alpha$ [39]. Upregulated CXCL12 expression in RA SMSCs may provide a helpful clue in elucidating the pathogenesis of and treating RA. IL6 is an important proinflammatory cytokine of RA that is involved in leukocyte activation, antibody production, and some systemic symptoms, such as asthenia, acutephase mediator response, and anemia [40]. Moreover, IL6 has successfully been targeted in RA therapies [41]. Increased IL6 expression in RA SMSCs is helpful for demonstrating the important role of RA SMSCs in RA pathogenesis.

The p53 signaling pathway was downregulated in RA SMSCs, with 13 differentially expressed genes revealed by the whole-genome expression analysis. Moreover, the expression of the tumor suppressor p53, which has been reported to play an important role in mediating cellular stress responses and is involved in cell cycle arrest, metabolism, senescence, DNA repair, and importantly proapoptosis [42], was significantly 
lower in RA SMSCs compared to HD SMSCs. Downstream genes of p53, such as BBC3 [43], CASP9 [44], GADD45B [45], IGF1 [46], and IGFBP3 [47], have also been reported to play roles in certain proapoptotic effects. In our study, the abovementioned genes were also downregulated in RA SMSCs, suggesting that RA SMSCs might have a stronger resistance to apoptosis. The apoptosis of transplanted MSCs has limited their therapeutic potential thus far [24]. As such, MSCs with a stronger resistance to apoptosis could demonstrate improved posttransplantation survival, which may be another advantage for autologous SMSC transplantation in RA patients.

\section{Conclusion}

In the present study, we screened differentially expressed genes of RA SMSCs and HD SMSCs and investigated them with GO and KEGG pathway analyses. We analyzed significantly enriched terms of differentially expressed genes in the biological process, cellular component, and molecular function domains. Moreover, we found that the MAPK signaling and rheumatoid arthritis pathways were upregulated and the p53 signaling pathway was downregulated in the RA SMSCs. All these data provide clues regarding the pathogenesis of RA and could facilitate therapeutic applications of RA SMSCs. Finally, future work will validate these differentially expressed genes and investigate both RA etiologies and potential therapeutic strategies.

\section{Competing Interests}

The authors declare that there is no conflict of interests regarding the publication of this paper.

\section{Authors' Contributions}

Jingyi Hou and Yi Ouyang contributed equally to this work and are the co-first authors. Weiping Li and Rui Yang contributed equally to this work and are the co-corresponding authors.

\section{Acknowledgments}

The authors appreciate the sincere guidance in their work from Yilong Education. This study was financially supported by the National Natural Science Foundation of China (no. 81472102) and Guangdong Natural Science Foundation (no. 2015A030313085).

\section{References}

[1] D. L. Scott, F. Wolfe, and T. W. J. Huizinga, "Rheumatoid arthritis," The Lancet, vol. 376, no. 9746, pp. 1094-1108, 2010.

[2] E. Zampeli, P. G. Vlachoyiannopoulos, and A. G. Tzioufas, "Treatment of rheumatoid arthritis: unraveling the conundrum," Journal of Autoimmunity, vol. 65, pp. 1-18, 2015.

[3] R. S. Woodrick and E. M. Ruderman, "Safety of biologic therapy in rheumatoid arthritis," Nature Reviews Rheumatology, vol. 7, no. 11, pp. 639-652, 2011.
[4] F. Barry and M. Murphy, "Mesenchymal stem cells in joint disease and repair," Nature Reviews Rheumatology, vol. 9, no. 10, pp. 584-594, 2013.

[5] Y. Shi, G. Hu, J. Su et al., "Mesenchymal stem cells: a new strategy for immunosuppression and tissue repair," Cell Research, vol. 20, no. 5, pp. 510-518, 2010.

[6] Y. Sakaguchi, I. Sekiya, K. Yagishita, and T. Muneta, "Comparison of human stem cells derived from various mesenchymal tissues: superiority of synovium as a cell source," Arthritis and Rheumatism, vol. 52, no. 8, pp. 2521-2529, 2005.

[7] L. Zangi, R. Margalit, S. Reich-Zeliger et al., "Direct imaging of immune rejection and memory induction by allogeneic mesenchymal stromal cells," STEM CELLS, vol. 27, no. 11, pp. 2865-2874, 2009.

[8] A. J. Nauta, G. Westerhuis, A. B. Kruisselbrink, E. G. A. Lurvink, R. Willemze, and W. E. Fibbe, "Donor-derived mesenchymal stem cells are immunogenic in an allogeneic host and stimulate donor graft rejection in a nonmyeloablative setting," Blood, vol. 108, no. 6, pp. 2114-2120, 2006.

[9] S. Dhingra, P. Li, X.-P. Huang et al., "Preserving prostaglandin E2 level prevents rejection of implanted allogeneic mesenchymal stem cells and restores postinfarction ventricular function," Circulation, vol. 128, no. 11, supplement 1, pp. S69-S78, 2013.

[10] F. C. Arnett, S. M. Edworthy, D. A. Bloch et al., "The American Rheumatism Association 1987 revised criteria for the classification of rheumatoid arthritis," Arthritis and Rheumatism, vol. 31, no. 3, pp. 315-324, 1988.

[11] C. De Bari, F. Dell'Accio, P. Tylzanowski, and F. P. Luyten, "Multipotent mesenchymal stem cells from adult human synovial membrane," Arthritis and Rheumatism, vol. 44, no. 8, pp. 19281942, 2001.

[12] M. Dominici, K. Le Blanc, I. Mueller et al., "Minimal criteria for defining multipotent mesenchymal stromal cells. The International Society for Cellular Therapy position statement," Cytotherapy, vol. 8, no. 4, pp. 315-317, 2006.

[13] Y. Wu, M. Ren, R. Yang et al., "Reduced immunomodulation potential of bone marrow-derived mesenchymal stem cells induced CCR $4^{+} \mathrm{CCR}^{+}{ }^{+} \mathrm{Th} /$ Treg cell subset imbalance in ankylosing spondylitis," Arthritis Research \& Therapy, vol. 13, no. 1, article R29, 2011.

[14] P. Wang, Y. Li, L. Huang et al., "Effects and safety of allogenic mesenchymal stem cell intravenous infusion in active ankylosing spondylitis patients who failed NSAIDs: a 20-week clinical trial," Cell Transplantation, vol. 23, no. 10, pp. 1293-1303, 2014.

[15] Z. Xie, P. Wang, Y. Li et al., "Imbalance between bone morphogenetic protein 2 and noggin induces abnormal osteogenic differentiation of mesenchymal stem cells in ankylosing spondylitis," Arthritis and Rheumatology, vol. 68, no. 2, pp. 430-440, 2016.

[16] Y. Li, P. Wang, Z. Xie et al., "Whole genome expression profiling and signal pathway screening of MSCs in ankylosing spondylitis," Stem Cells International, vol. 2014, Article ID 913050, 11 pages, 2014.

[17] D. van der Woude, J. J. Houwing-Duistermaat, R. E. M. Toes et al., "Quantitative heritability of anti-citrullinated protein antibody-positive and anti-citrullinated protein antibodynegative rheumatoid arthritis," Arthritis and Rheumatism, vol. 60, no. 4, pp. 916-923, 2009.

[18] T. Yoshizawa, D. Hammaker, S. E. Sweeney, D. L. Boyle, and G. S. Firestein, "Synoviocyte innate immune responses: I. Differential regulation of interferon responses and the JNK pathway by 
MAPK kinases," Journal of Immunology, vol. 181, no. 5, pp. 32523258, 2008.

[19] C.-H. Li, L.-L. Xu, J.-X. Zhao et al., "CXCL16 upregulates RANKL expression in rheumatoid arthritis synovial fibroblasts through the JAK2/STAT3 and p38/MAPK signaling pathway," Inflammation Research, vol. 65, no. 3, pp. 193-202, 2016.

[20] L. Zhang, J. Luo, H. Wen, T. Zhang, X. Zuo, and X. Li, "MDM2 promotes rheumatoid arthritis via activation of MAPK and NF- $\kappa$ B," International Immunopharmacology, vol. 30, pp. 69-73, 2016.

[21] G. X. Liu, J. C. Zhu, X. Y. Chen et al., "Inhibition of adipogenic differentiation of bone marrow mesenchymal stem cells by erythropoietin via activating ERK and P38 MAPK," Genetics and Molecular Research, vol. 14, no. 2, pp. 6968-6977, 2015.

[22] M. Leyva-Leyva, A. López-Diáz, L. Barrera et al., "Differential expression of adhesion-related proteins and MAPK pathways lead to suitable osteoblast differentiation of human mesenchymal stem cells subpopulations," Stem Cells and Development, vol. 24, no. 21, pp. 2577-2590, 2015.

[23] S. L. Tomchuck, K. J. Zwezdaryk, S. B. Coffelt, R. S. Waterman, E. S. Danka, and A. B. Scandurro, "Toll-like receptors on human mesenchymal stem cells drive their migration and immunomodulating responses," STEM CELLS, vol. 26, no. 1, pp. 99-107, 2008.

[24] X. Zeng, S. P. Yu, T. Taylor, M. Ogle, and L. Wei, "Protective effect of apelin on cultured rat bone marrow mesenchymal stem cells against apoptosis," Stem Cell Research, vol. 8, no. 3, pp. 357367, 2012.

[25] Y. Shi, Y. Feng, J. Kang et al., "Critical regulation of CD4 ${ }^{+}$ T cell survival and autoimmunity by $\beta$-arrestin 1," Nature Immunology, vol. 8, no. 8, pp. 817-824, 2007.

[26] D.-H. Lee, E. Geyer, A.-C. Flach et al., "Central nervous system rather than immune cell-derived BDNF mediates axonal protective effects early in autoimmune demyelination," Acta Neuropathologica, vol. 123, no. 2, pp. 247-258, 2012.

[27] R. Hohlfeld, M. Kerschensteiner, C. Stadelmann, H. Lassmann, and H. Wekerle, "The neuroprotective effect of inflammation: implications for the therapy of multiple sclerosis," Neurological Sciences, vol. 27, supplement 1, pp. s1-s7, 2006.

[28] C. Weidler, C. Holzer, M. Harbux et al., "Low density of sympathetic nerve fibres and increased density of brain derived neurotrophic factor positive cells in RA synovium," Annals of the Rheumatic Diseases, vol. 64, no. 1, pp. 13-20, 2005.

[29] D. Clemente, M. C. Ortega, F. J. Arenzana, and F. de Castro, "FGF-2 and anosmin-1 are selectively expressed in different types of multiple sclerosis lesions," The Journal of Neuroscience, vol. 31, no. 42, pp. 14899-14909, 2011.

[30] S. A. Chung, E. E. Brown, A. H. Williams et al., "Lupus nephritis susceptibility loci in women with systemic lupus erythematosus," Journal of the American Society of Nephrology, vol. 25, no. 12, pp. 2859-2870, 2014.

[31] L. Liu, Y. Wang, H. Fan et al., "MicroRNA-181a regulates local immune balance by inhibiting proliferation and immunosuppressive properties of mesenchymal stem cells," STEM CELLS, vol. 30, no. 8, pp. 1756-1770, 2012.

[32] T. Maruyama, A. J. Mirando, C.-X. Deng, and W. Hsu, "The balance of WNT and FGF signaling influences mesenchymal stem cell fate during skeletal development," Science Signaling, vol. 3, no. 123, Article ID ra40, 2010.

[33] X. Guan, Y. Gao, J. Zhou et al., "MiR-223 regulates adipogenic and osteogenic differentiation of mesenchymal stem cells through a C/EBPs/miR-223/FGFR2 regulatory feedback loop," STEM CELLS, vol. 33, no. 5, pp. 1589-1600, 2015.

[34] J. Zhang, J. C. Ho, Y. C. Chan, Q. Lian, C. Siu, and H. Tse, “Overexpression of myocardin induces partial transdifferentiation of human-induced pluripotent stem cell-derived mesenchymal stem cells into cardiomyocytes," Physiological Reports, vol. 2, no. 2, Article ID e00237, 2014.

[35] M. E. Babadagli, B. Tezcan, S. T. Yilmaz, and A. C. Tufan, "Matrilin-3 as a putative effector of C-type natriuretic peptide signaling during TGF- $\beta$ induced chondrogenic differentiation of mesenchymal stem cells," Molecular Biology Reports, vol. 41, no. 9, pp. 5549-5555, 2014.

[36] J. R. Glossop and S. H. Cartmell, "Effect of fluid flow-induced shear stress on human mesenchymal stem cells: differential gene expression of IL1B and MAP3K8 in MAPK signaling," Gene Expression Patterns, vol. 9, no. 5, pp. 381-388, 2009.

[37] X. Wu, S. A. Estwick, S. Chen et al., "Neurofibromin plays a critical role in modulating osteoblast differentiation of mesenchymal stem/progenitor cells," Human Molecular Genetics, vol. 15, no. 19, pp. 2837-2845, 2006.

[38] Y. Liu, E. G. Shepherd, and L. D. Nelin, "MAPK phosphatasesregulating the immune response," Nature Reviews Immunology, vol. 7, no. 3, pp. 202-212, 2007.

[39] D. L. Asquith, S. A. Bryce, and R. J. B. Nibbs, "Targeting cell migration in rheumatoid arthritis," Current Opinion in Rheumatology, vol. 27, no. 2, pp. 204-211, 2015.

[40] M.-C. Boissier, L. Semerano, S. Challal, N. SaidenbergKermanac'h, and G. Falgarone, "Rheumatoid arthritis: from autoimmunity to synovitis and joint destruction," Journal of Autoimmunity, vol. 39, no. 3, pp. 222-228, 2012.

[41] S. Siebert, A. Tsoukas, J. Robertson, and I. McInnes, "Cytokines as therapeutic targets in rheumatoid arthritis and other inflammatory diseases," Pharmacological Reviews, vol. 67, no. 2, pp. 280-309, 2015.

[42] K. K. Hoe, C. S. Verma, and D. P. Lane, "Drugging the p53 pathway: understanding the route to clinical efficacy," Nature Reviews Drug Discovery, vol. 13, no. 3, pp. 217-236, 2014.

[43] L. Vucicevic, M. Misirkic-Marjanovic, V. Paunovic et al., "Autophagy inhibition uncovers the neurotoxic action of the antipsychotic drug olanzapine," Autophagy, vol. 10, no. 12, pp. 2362-2378, 2014.

[44] E. P. Lu, M. McLellan, L. Ding et al., "Caspase-9 is required for normal hematopoietic development and protection from alkylator-induced DNA damage in mice," Blood, vol. 124, no. 26, pp. 3887-3895, 2014.

[45] Z. Chen, X. Wan, Q. Hou et al., "GADD45B mediates podocyte injury in zebrafish by activating the ROS-GADD45B-p38 pathway," Cell Death \& Disease, vol. 7, no. 1, Article ID e2068, 2016.

[46] S. Cavallaro, "Cracking the code of neuronal apoptosis and survival," Cell Death and Disease, vol. 6, Article ID e1963, 2015.

[47] H. Sun, D. C. Lin, Q. Cao et al., "CRM1 inhibition promotes cytotoxicity in ewing sarcoma cells by repressing EWS-FLI1Dependent IGF-1 Signaling," Cancer Research, vol. 76, no. 9, pp. 2687-2697, 2016. 



Submit your manuscripts at

http://www.hindawi.com
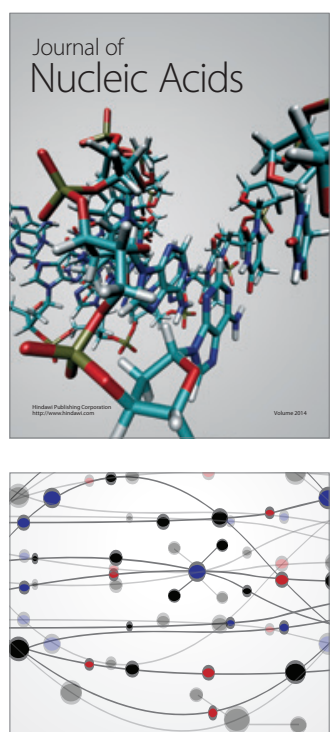

The Scientific World Journal
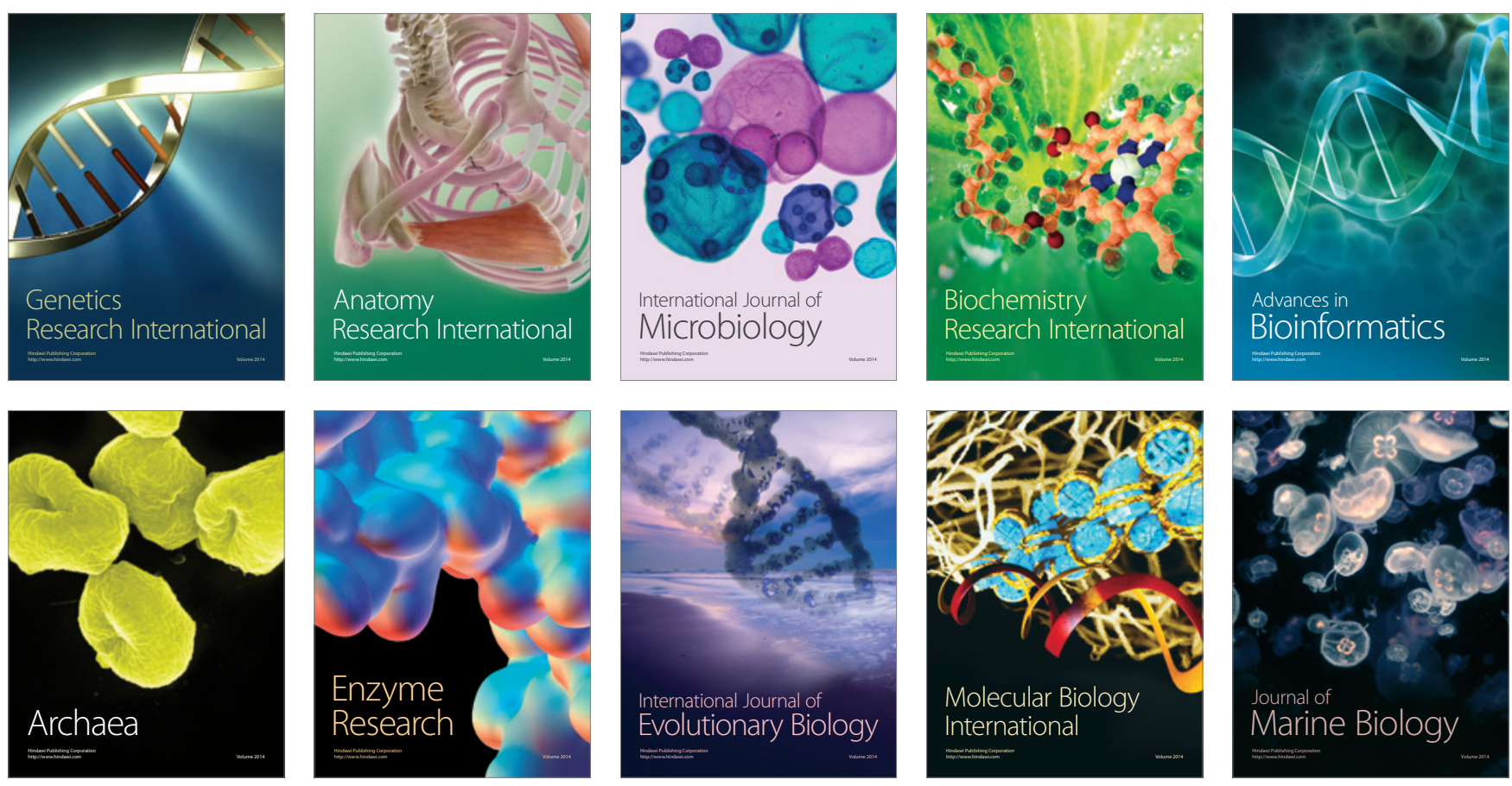